\title{
Facilitation versus inhibition in the masked priming same-different matching task
}

\author{
Manuel Perea ${ }^{1}$, Carmen Moret-Tatay ${ }^{1,2}$, and Manuel Carreiras ${ }^{3,4}$ \\ ${ }^{1}$ Departamento de Metodología, Universitat de València, Valencia, Spain \\ ${ }^{2}$ Departamento de Metodología, Psicología Básica, y Psicología Social, Universidad Católica de Valencia, \\ Valencia, Spain \\ ${ }^{3}$ Basque Center for Cognition, Brain, and Language, Donostia-San Sebastián, Spain \\ ${ }^{4}$ IKERBASQUE, Basque Foundation for Science, Bilbao, Spain
}

In the past years, growing attention has been devoted to the masked priming same-different task introduced by Norris and Kinoshita (2008, Journal of Experimental Psychology: General). However, a number of researchers have raised concerns on the nature of the cognitive processes underlying this task-in particular the suspicion that masked priming effects in this task are mostly inhibitory in nature and may be affected by probe-prime contingency. To examine the pattern of facilitative/inhibitory priming effects in this task, we conducted two experiments with an incremental priming paradigm using four stimulus-onset asynchronies (13, 27, 40, and $53 \mathrm{~ms})$. Experiment 1 was conducted under a predictive-contingency scenario (probe-prime-target; i.e., "same" trials: HOUSE-house-HOUSE vs. housewater-HOUSE; "different" trials: field-house-HOUSE vs. field-water-HOUSE), while Experiment 2 employed a zero-contingency scenario (i.e., "same" trials: HOUSE-house-HOUSE vs. house-waterHOUSE; "different" trials: field-field-HOUSE vs. field-water-HOUSE). Results revealed that, for "same" responses, both facilitation and inhibition increased linearly with prime duration in the two scenarios, whereas the pattern of data varied for "different" responses, as predicted by the Bayesian Reader model.

Keywords: Masked priming; Incremental priming; Word recognition.

In the past years, an increasing number of studies have employed the masked priming samedifferent matching task introduced by Norris and Kinoshita (2008; e.g., Duñabeitia, Kinoshita, Carreiras, \& Norris, 2011; García-Orza \& Perea, 2011; García-Orza, Perea, \& Muñoz, 2010; Kinoshita, Castles, \& Davis, 2009; Kinoshita \&
Kaplan, 2008; Kinoshita \& Lagoutaris, 2010; Kinoshita \& Norris, 2009, 2010, 2011; Perea, Abu Mallouh, García-Orza, \& Carreiras, 2011; Perea \& Acha, 2009; Perea, Duñabeitia, Pollatsek, \& Carreiras, 2009). A depiction of the task is presented in Figure 1. The participant's task is to decide whether or not a probe and a subsequently presented

Correspondence should be addressed to Manuel Perea, Departamento de Metodología, Facultad de Psicología, Av. Blasco Ibáñez, 21, 46010-Valencia, Spain. E-mail: mperea@valencia.edu

This research has been partially supported by Grants PSI2009-08889/PSIC, PSI2008-04069/PSIC, and CONSOLIDERINGENIO2010 CSD2008-00048 from the Spanish Government. We would like to thank two anonymous reviewers for their comments on a previous version of the present paper. 

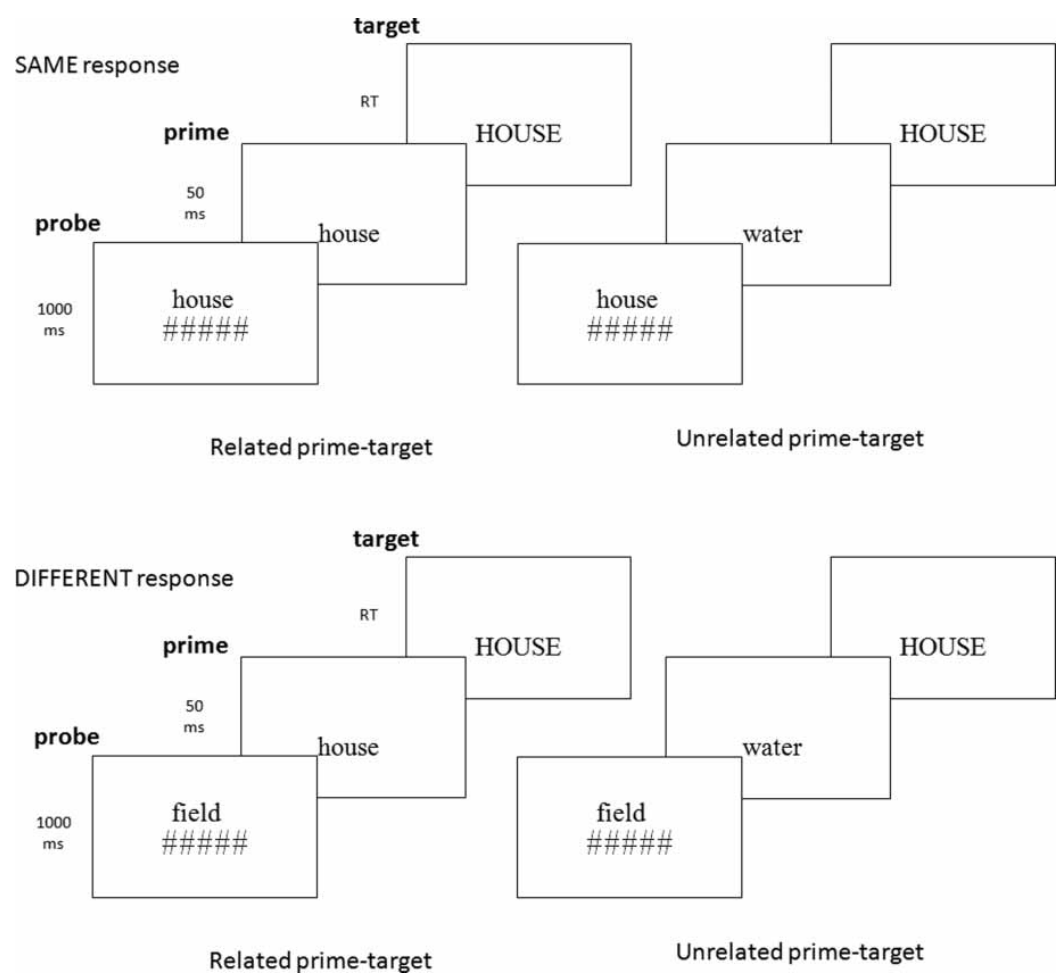

Figure 1. Depiction of the masked priming same-different task: A probe (in lower case) is presented for 1,000 ms, together with a forward mask (\#\#\#\#) for the subsequent prime. Then a lower-case prime is presented for $50 \mathrm{~ms}$ and is immediately replaced by an upper-case target stimulus until the participant's response.

target (i.e., a comparison string) are the same. A forwardly masked prime is presented in the same location as the target for a brief duration (around $50 \mathrm{~ms}$ ). For "same" responses, targets preceded by a related prime are responded to faster than the targets preceded by an unrelated prime (probeprime-TARGET; e.g., house-house-HOUSE faster than house-water-HOUSE), whereas there are no priming effects for "different" responses (i.e., no differences between field-house-HOUSE and field-water-HOUSE; see Norris \& Kinoshita, 2008). One advantage of this task, relative to lexical decision or naming, is its enormous versatility: It can be applied to a large variety of stimuli (e.g., words, symbols, letters, pseudoletters, objects, etc.; see García-Orza \& Perea, 2011; García-Orza et al., 2010; Kinoshita \& Lagoutaris, 2010; Perea et al., 2009).
Norris and Kinoshita (2008, 2010) offered a detailed explanation of the masked priming effects with the same-different task in the framework of the Bayesian Reader model. The general logic is the following: For "same" trials (househouse-HOUSE vs. house-water-HOUSE), the related prime house generates evidence supporting the hypothesis that the target stimulus is the same as the probe, whereas the unrelated prime water generates evidence supporting the hypothesis that the target is different to the probe. Thus, the Bayesian Reader model predicts a masked priming effect for "same" trials. For "different" trials (e.g., field-house-HOUSE vs. field-waterHOUSE), both the related prime house and the unrelated prime water generate similar evidence supporting the hypothesis that the target is different from the probe. Therefore, for "different" trials, the 
Bayesian Reader model does not predict a masked priming effect. Indeed, simulations on the model confirmed these predictions (see Norris \& Kinoshita, 2008, Figure 4).

In the present study, we put to test two issues raised in papers, reviews, and/or conference presentations that cast some doubts on the usefulness of the masked priming same-different matching task. The first concern is the facilitative versus inhibitory nature of masked priming effects in the task. The second concern is whether or not a potential confound in the design of the usual masked priming same-different experiments can modulate the magnitude of priming effects.

\section{Are masked priming effects in the same- different task inhibitory in nature?}

A number of critics of the masked priming samedifferent task have argued-mostly at conferences and/or reviews - that the obtained priming effects are inherently inhibitory. Here is the logic flow from the critics - in terms of an activation-based model: For "same" trials, the target is already strongly activated by the probe. Thus, a matching prime would presumably provide a modest additional activation (i.e., small facilitation), while a prime that is inconsistent with the target might begin to induce a "different" response, which must be overridden (i.e., a strong inhibitory effect). In other words, a prime that is inconsistent with the target may slow down reaction times, relative to a baseline condition, more than a prime consistent with the target speeds reaction times.

Therefore, the presence of a robust inhibitory (rather than facilitative) masked priming effect in the same-different task would clearly pose some problems for the Bayesian Reader account of the masked priming same-different task. Prior work with the masked priming same-different task has focused on across-condition comparisons (i.e., unrelated priming condition vs. related priming condition), and these comparisons cannot inform us of the facilitative/inhibitory nature of the observed priming effects.
Probe-contingency versus zero-contingency experiments: Does contingency matter?

In the design employed in the usual masked priming same-different experiments (including the Norris \& Kinoshita, 2008, experiments), there is a potential confound: The prime is manipulated with respect to the target (see Figure 1). This implies that "different" trials involve probes that are always different from the primes (e.g., fieldhouse-HOUSE vs. field-water-HOUSE). That is, if probe and prime are the same, the response is necessarily "same", whereas if probe and prime are different, the target may be the same or different from the probe.

Under these circumstances, a number of critics have argued that participants may be preparing their response on the basis of probe-prime relatedness (e.g., "if probe and target look similar, prepare a 'same' response") rather than on the basis of prime-target relatedness. This is the so-called "response bias" (or "implicit learning") account (see Kinoshita \& Norris, 2010, and Norris \& Kinoshita, 2010, for descriptions of this account; see also Bowers, 2010a, 2010b, for criticism of the Bayesian Reader model). The Bayesian Reader model makes again a quite strong prediction in this respect. In a masked priming paradigm, the model "predicts that priming for the same response should be unaffected by the contingency" (Kinoshita \& Norris, 2010, p. 198). This is so because one basic tenet of the Bayesian Reader model is that a given masked prime is not processed as a separate perceptual event from the target-as a result, participants cannot prepare a response depending on the probe-prime relationship (see Norris \& Kinoshita, 2008).

Recently, Perea and Acha (2009) and Kinoshita and Norris (2010) avoided the above-cited confound by manipulating not the relationship between prime and target, but the relationship between probe and prime (i.e., a zero-contingency experiment; see also Kinoshita \& Kaplan, 2008, for a similar experiment with single letters). For "same" trials, the comparison was exactly the same as that in previous experiments (e.g., house-house-HOUSE vs. house-water-HOUSE), 
whereas for "different" trials, the comparison was between trials like field-field-HOUSE versus field-water-HOUSE. That is, if the probe and prime are the same, in half of the trials the responses will be "same" responses (e.g., househouse-HOUSE), and in the other half they will be "different" responses (e.g., field-fieldHOUSE); in addition, if the probe and prime are different, in half of the trials the responses will be "same" responses (e.g., house-waterHOUSE), and in the other half they will be "different" responses (e.g., house-water-HOUSE). This way, participants cannot diagnostically use the probe-prime relationship to prepare a response. A “response bias" account would predict a greater masked priming effect in (standard) predictivecontingency experiments than in zero-contingency experiments: The reason is that in a zerocontingency experiment there is no advantage of the prime being diagnostic of the response, whereas in a predictive-contingency experiment when the probe and the prime look similar, the response is always "same". Alternatively, the Bayesian Reader model predicts no differences under predictive versus zero-contingency scenarios.

For "same" trials, the results from the zero-contingency experiments of Perea and Acha (2009), Kinoshita and Kaplan (2008), and Kinoshita and Norris (2010) were remarkably similar to the results using "standard" (predictive-contingency) conditions, thus reinforcing the Bayesian Reader account. For "different" responses, response times were higher (and error rates higher) in the related condition than in the unrelated condition (see Kinoshita \& Kaplan, 2008; Kinoshita \& Norris, 2010; Perea \& Acha, 2009), although the effect only reached the classical criterion for significance in the single letter experiment of Kinoshita and Kaplan (2008). This inhibitory trend can also be easily explained by the Bayesian Reader model: Under a zero-contingency scenario, in the sequence field-field-HOUSE, the prime field is the same as the probe; this means that the prime field generates evidence supporting a "same" response. Given that the correct response is "different", there may be some processing cost associated at overriding the evidence supporting "same" (i.e., longer reaction times and/or more errors).
The findings from Perea and Acha (2009) and Kinoshita and Norris (2010) suggest that the pattern of "same" responses is similar regardless of the probe-prime contingency in the masked priming technique - as predicted by the Bayesian Reader model. Nonetheless, we believe that it is important to examine not only whether the across-condition priming effects (i.e., the difference between the unrelated and related conditions) are similar under the two scenarios, but also to examine the time course of facilitative/inhibitory effects under these two scenarios. This would provide a much more constraining test of the Bayesian Reader model. Furthermore, it is also relevant to explore in further detail the pattern of facilitative/inhibitory priming effects for "different" responses-note that the two previous studies in this issue with word stimuli reported a nonsignificant inhibitory trend.

One final note: When the prime is processed as a different perceptual object, as is the case of unmasked visible primes, then prime diagnosticity influences responses with visible primes. In a recent experiment, Kinoshita and Norris (2010) found a large repetition priming effect $(104 \mathrm{~ms})$ for "same" trials under a predictive-contingency scenario, while the parallel effect under a zero-contingency scenario was a nonsignificant 14-ms effect. For "different" trials, Kinoshita and Norris found much slower $(60 \mathrm{~ms})$ response times in the identity condition than in the unrelated condition (e.g., field-house-HOUSE slower than field-waterHOUSE) under the predictive-contingency scenario, whereas they found a repetition priming effect (24 ms) under the zero-contingency scenario (e.g., field-field-HOUSE faster than field-waterHOUSE). Interestingly, as mentioned above, Kinoshita and Norris (2010) did not find an effect of prime diagnosticity when the primes were presented masked, which is consistent with the view that "the [masked] prime is not processed as a separate event from the target" (p. 198).

\section{Rationale of the experiments}

The aim of the present experiments is to examine two critical, methodological issues concerning the 
masked priming same-different task: (a) to what degree the obtained effects are facilitative or inhibitory, and (b) to what degree the pattern of facilitative/inhibitory effects is modulated by probe-prime contingency.

One highly valuable tool to study the time course of facilitative/inhibitory priming effects is the incremental priming technique (Jacobs, Grainger, \& Ferrand, 1995; see also Frost, Ahissar, Gottesman, \& Tayeb, 2003; Giraudo \& Grainger, 2001; Grainger \& Frenck-Mestre, 1998; Peressotti \& Grainger, 1999; Ziegler, Ferrand, Jacobs, Rey, \& Grainger, 2000). In an incremental priming experiment, the critical manipulation is not just the prime-target relationship (e.g., related vs. unrelated priming conditions; i.e., across-condition comparisons), but also the prime exposure duration (or, in other studies, the intensity of the stimuli; i.e., within-condition comparisons). The idea is to use a very brief prime duration (e.g., $13 \mathrm{~ms}$ ) at which masked priming effects are negligible. This condition will play the role of within-condition baseline. This within-condition baseline will be used against other prime exposure durations (e.g., 27, 40, $53 \mathrm{~ms})$. The raison d'être of the technique, as described by Ziegler et al. (2000), is straightforward: "If performance improves with respect to this within-condition baseline, then the prime has facilitated target processing. If performance decreases, then the prime has inhibited target processing." (p. 673).

\section{The facilitative versus inhibitory nature of masked priming effects}

Prior research with the masked priming lexical decision task has shown that, relative to the within-condition baseline, masked repetition priming effects are a mixture of facilitative and inhibitory priming effects - with facilitatory effects being greater than inhibitory effects. For instance, Giraudo and Grainger (2001, Experiment 1) found that response times for the repetition priming condition were $28 \mathrm{~ms}$ faster at the $57-\mathrm{ms}$ stimulus-onset asynchrony (SOA) than at the 14-ms SOA (i.e., the within-condition baseline), whereas response times for the unrelated priming condition were $18.5 \mathrm{~ms}$ slower at the $57-\mathrm{ms} \mathrm{SOA}$ than at the $14-\mathrm{ms} \mathrm{SOA}^{1}$

What about the masked priming same-different task? The fact that the target has already been activated by the probe in the same-different task may suggest that the same-different task leads to mostly inhibitory priming effects. As indicated earlier, some researchers have argued that, for "same" trials, a related prime may provide a small additional activation (i.e., an insignificant facilitation), whereas a prime that is inconsistent with the target might induce a "different" response (i.e., a robust inhibition). However, the Bayesian Reader model would predict a mixture of facilitative and inhibitory priming effects: For "same" responses, a related prime provides positive evidence supporting a "same" response (i.e., a facilitation effect), whereas an unrelated prime provides evidence supporting a "different" response (i.e., an inhibitory effect). (We should note here that Norris and Kinoshita, 2008, do not use the term "activation" in describing the operation of the Bayesian Reader model.)

\section{Predictive versus zero probe/prime contingency}

The rationale for the manipulation of the predictive versus zero probe/prime contingency is to perform a stringent test of the "response bias" account versus the Bayesian Reader account. In the "response bias" account (see Kinoshita \& Norris, 2010), under the predictive-contingency scenario, if probe and prime are the same, the response is "same" (see Figure 1), whereas if probe and prime are different, the target is likely to be different from the target (e.g., 2 out of 3 times in the design of Figure 1). That is, the obtained masked priming effects may pertain to the relationship between the probe and the prime, rather than to the relationship between the prime and the target. Under a zero-contingency scenario, the probe-prime relationship is no longer diagnostic of the responses to the target. Thus, if

\footnotetext{
${ }^{1}$ In Giraudo and Grainger's (2001) Experiment 1, they used a 0-ms prime as the within-condition baseline. However, for comparison purposes, we have employed the 14-ms SOA condition because it is closer to the one employed in the present experiments.
} 
participants prepare their responses on the basis of the implicit learning of probe-prime relationship, the pattern of priming effects for "same" responses would differ under predictive-contingency and zero-contingency scenarios (see Kinoshita \& Norris, 2010). Alternatively, the Bayesian Reader model predicts that "same" responses should be unaltered by probe-prime contingency.

\section{The experiments}

We conducted two experiments using a masked priming same-different judgement task. To assess the time course of the facilitative/inhibitory priming effects in this task, we employed an incremental priming technique-the prime exposure durations were 13, 27, 40, and $53 \mathrm{~ms}$. The briefest prime duration (13 $\mathrm{ms}$ ) will presumably fail to reveal any significant priming effects, whereas the longest prime duration (53 ms) is virtually the same as that in prior work with this task (i.e., $50 \mathrm{~ms}$ ). As in previous work by Grainger and colleagues (e.g., Grainger \& Frenck-Mestre, 1998; Jacobs et al., 1995; Peressotti \& Grainger, 1999; Ziegler et al., 2000), we followed a psychophysical approach: A small sample of highly trained participants undertook several test sessions. Experiment 1 was a masked repetition priming experiment under "standard" (i.e., predictive-contingency) conditions (e.g., "same" trials: HOUSE-house-HOUSE vs. house-waterHOUSE; "different" trials: field-house-HOUSE vs. field-water-HOUSE). Thus, the prime was a diagnostic of the response to the target stimulus. Experiment 2 was the same as Experiment 1, except that it was conducted under zerocontingency conditions (e.g., "same" trials: HOUSE-house-HOUSE vs. house-waterHOUSE; "different" trials: field-field-HOUSE vs. field-water-HOUSE).

The same-different task is thought to tap orthographic rather than lexical processing. Indeed, prior studies have shown that pattern of masked priming effects is very similar for words, pronounceable pseudowords, and consonant strings (Norris \& Kinoshita, 2008; see also García-Orza et al., 2010; Perea \& Acha, 2009). To further examine whether the processes of facilitation/inhibition in the masked priming same-different task may be modulated by lexical status, we employed both word trials and nonword trials. Keep in mind that a difference in the pattern of priming effects with words versus nonwords would go against the Bayesian Reader model.

\section{EXPERIMENT 1}

\section{Method}

\section{Participants}

Four well-trained individuals from the laboratory in Valencia took part in the experiment. All of them were native of Spanish and had normal (or corrected-to-normal) vision.

\section{Materials}

The targets were 320 Spanish words of five to eight letters and 320 pronounceable nonwords of five to eight letters. The mean word frequency per million of the word stimuli was 38 , and the mean Coltheart's $N$ was 0.5 (Davis \& Perea, 2005). For the word stimuli, a list of 320 unrelated prime words matched in length to the target stimuli-on a pairwise basis-was also generated to serve as unrelated primes. For the purposes of the same-different task, we also created a list of 320 additional words. The target was presented in uppercase and was preceded by a prime that was (a) the same as the target (related condition), or (b) a word unrelated to the target (unrelated condition). For each target (e.g., OTONO; the Spanish for Autumn), we had a related prime (otoño) and an unrelated prime (arena; the Spanish for sand) - the two potential probes for this target were otoño ("same" response) and falda ("different" response; falda is the Spanish for skirt). Given that participants might somehow learn the association of (probe-TARGET) otoño-OTONO or falda-OTONO across several sessions, we tried to make this association more difficult by creating a parallel list in which the target was $F A L D A$, the related prime was falda, and the unrelated prime was arena-the two potential probes for this target were falda ("same" 
response) and otoño ("different" response), as in (probe-TARGET) falda-FALDA or otoño$F A L D A$. In addition, for the nonword stimuli, a list of 320 unrelated prime nonwords matched in length to the target stimuli-on a pairwise basis-was also generated to serve as unrelated primes. For the purposes of the same-different task, we also created a list of 320 additional nonwords. (All the nonwords in the experiment were orthographically legal and pronounceable; they had been created by replacing two/three letters from real Spanish words that did not form part of the experimental set.) The creation of the nonword pairs was parallel to that of the word pairs (see above). The computer selected randomly a set of 320 related pairs (160 word pairs and 160 nonword pairs) and a set of 320 unrelated pairs (160 word pairs and 160 nonword pairs) for each individual/session. Each participant received a different random sample of pairs. The complete set of materials is available at http://www.uv.es/ mperea/incremental_same.pdf. The factors in the experiment were prime-target relationship (related, unrelated), stimulus onset asynchrony (13, 27, 40, $53 \mathrm{~ms}$ ), lexical status (word, nonword), and type of response (same, different). On each session, the participants received 20 trials per condition. All these factors were varied within the same block. Each participant took part in four sessions.

\section{Procedure}

Participants were tested individually in a quiet room. The stimuli were presented using a PC running the DMDX software for Windows (Forster \& Forster, 2003) on a CRT monitor with a $13.3-\mathrm{ms}(75-\mathrm{Hz})$ refresh rate. In each trial, a reference string (i.e., probe) printed in lower case was presented above a forward mask consisting of a series of hash marks (\#s) for 1,000 ms. (The mask was “\#\#\#\#” for 5-letter stimuli and "\#\#\#\#\#\#" for 8-letter stimuli.) Next, the probe disappeared, and the forward mask was replaced by a lower-case prime for 13 , 26,40 , or $53 \mathrm{~ms}$, which in turn was replaced by a target presented in upper case. (Note that "prime duration" and SOA were exactly the same in the present study.) The target stimulus remained on the screen until the participant's response. Participants were told that they would see two strings of letters (i.e., a probe and a target), and that they were to press the " $\mathrm{M}$ " button if they thought the probe target were the same stimulus and the " $Z$ " button if they thought the probe and target were a different stimulus. Participants were instructed to make this decision as fast and as accurately as possible. Participants reported not having seen any prime stimuli when asked after the experiment. Each participant received a different, randomized order of trials across the four sessions (640 trials on each session). The total number of experimental trials per participant was 2,560. Before each experimental block, there were 20 practice trials with the same manipulation as that in the experimental trials. Each experimental session lasted for around 20-25 $\mathrm{min}$.

\section{Results and discussion}

Incorrect responses (3.1\% of trials) and response times (RTs) less than 250 or greater than $1,500 \mathrm{~ms}$ (less than $0.01 \%$ of trials) were excluded from the response time data. The mean latencies for correct responses and error rates are presented in Table 1. Given that each participant received a different random sample of pairs, there is no need to conduct analyses by items (see Perea \& Gotor, 1997, for a similar procedure).

Analyses of variance (ANOVAs) based on the participant response latencies and error rates were conducted on the basis of a 2 (prime type: related, unrelated $) \times 2$ (lexical status of probe/ target: word, nonword) $\times 2$ (type of response: same, different) $\times 4$ (SOA: 13, 27, 40, $53 \mathrm{~ms}$ ). We should note here that session was also included as a factor in the ANOVAs, but leaving aside a marginal main effect of session in the RT data, $F(3,9)=3.93, M S E=2,473, p=.048$, session did not interact with any of the other factors, all ps $>.30$ (i.e., the observed masked priming effects were similar in magnitude across sessions), and it is not considered further.

The ANOVA on the latency data showed that the Prime Type $\times$ SOA interaction was 
Table 1. Mean response times and percentage of errors for word and pseudoword targets in Experiment 1

\begin{tabular}{|c|c|c|c|c|c|c|}
\hline \multirow[b]{2}{*}{ Responses } & & & \multicolumn{4}{|c|}{$S O A$} \\
\hline & & & $13 \mathrm{~ms}$ & $27 \mathrm{~ms}$ & $40 \mathrm{~ms}$ & $53 \mathrm{~ms}$ \\
\hline \multirow[t]{6}{*}{ Same } & Words & Related & $406(0.3)$ & $401(0.9)$ & $385(0.3)$ & $379(0.6)$ \\
\hline & & Unrelated & $410(0.6)$ & $420(2.8)$ & $438(7.5)$ & $474(19.7)$ \\
\hline & & Priming & $4(0.3)$ & 19 (1.9) & 53 (1.6) & $94(19.1)$ \\
\hline & Nonwords & Related & $433(1.3)$ & $428(0.6)$ & $413(0.9)$ & $401(1.3)$ \\
\hline & & Unrelated & 435 (1.9) & $438(2.2)$ & $462(5.6)$ & $482(20.0)$ \\
\hline & & Priming & $2(0.6)$ & $10(0.0)$ & $49(4.7)$ & 81 (18.8) \\
\hline \multirow[t]{6}{*}{ Different } & Words & Related & $467(2.8)$ & $454(4.1)$ & $437(3.1)$ & $430(2.8)$ \\
\hline & & Unrelated & $465(2.8)$ & $452(2.8)$ & 439 (1.2) & $426(1.3)$ \\
\hline & & Priming & $-2(0.0)$ & $-2(-1.3)$ & $2(-1.9)$ & $-4(-1.6)$ \\
\hline & Nonwords & Related & $466(2.2)$ & $453(2.8)$ & 445 (1.9) & $436(0.6)$ \\
\hline & & Unrelated & 464 (1.6) & $449(0.0)$ & $440(1.3)$ & $431(0.6)$ \\
\hline & & Priming & $-2(-0.6)$ & $-4(-2.8)$ & $-4(-0.6)$ & $-5(0.0)$ \\
\hline
\end{tabular}

Note: Predictive-contingency scenario. $\mathrm{SOA}=$ stimulus onset asynchrony. Mean response times in ms. Percentages of errors in parentheses.

modulated by type of response: three-way interaction, $F(3,9)=51.90, M S E=52.8, p<.001$. For that reason, we analysed separately "same" and "different" trials-as is the case in prior studies with this task (e.g., see Norris \& Kinoshita, 2008). Importantly, lexicality did not interact with any of the other factors - that is, there was just a main effect of lexicality, $F(1,3)$ $=11.00, \quad M S E=74.1, \quad p<.05$, while the $F$ ratios corresponding to the interactions with lexicality were far away from significance. Therefore, for simplicity, we focus on prime type and SOA as factors, regardless of lexical status and session.

\section{Same responses}

The ANOVA on the latency data revealed a main effect of prime type, $F(1,3)=42.75, M S E=$ 623.4, $p<.01$, and SOA, $F(1,3)=10.39, M S E$ $=115.2, p<.005$. The interaction between prime type and SOA was significant, $F(3,9)=$ 113.61, $M S E=49.5, p<.001$. This interaction reflected that the effect of prime type was not significant at the 13-ms SOA, $F(1,3)=1.55, M S E$ $=52.2, p>.30$, whereas it was significant at the other SOAs: 27-ms SOA, $F(1,3)=13.88, M S E$ $=98.7, p<.05 ; 40-\mathrm{ms}$ SOA, $F(1,3)=29.23$,
$M S E=271.7, p<.01 ; 53-\mathrm{ms}$ SOA, $F(1,3)=$ 89.92, $M S E=349.4, p<.02$.

To reveal the presence of facilitative/inhibitory effects, it is critical to look at the within-condition priming analysis. For the related condition, there was a decreasing linear trend, $F(1,3)=18.80$, $M S E=138.8, p<.05$, while the quadratic component was not significant $(F<1)$. For the unrelated condition, there was an increasing linear trend, $F(1,3)=157.49, \quad M S E=106.5, \quad p<$ .005 , as well as a significant quadratic component, $F(1,3)=11.93, M S E=72.2, p<.05$.

The ANOVA on the error data revealed main effects of prime type, $F(1,3)=15.66, M S E=$ 50.0, $p<.05$, and SOA, $F(1,3)=13.41, M S E=$ 23.3, $p<.005$. As in the latency data, the interaction between prime type and SOA was significant, $F(3,9)=17.62, M S E=17.9, p<$ .001 . This interaction reflected that the effect of prime type was not significant at the 13-ms SOA $(F<1)$, whereas it was significant (or marginally significant) at the other SOAs: 27-ms SOA, $F(1,3)=10.37, \quad M S E=1.14, p<.05 ; 40-\mathrm{ms}$ SOA, $F(1,3)=8.15, M S E=20.1, p=.065$; 53ms SOA, $F(1,3)=19.05, M S E=81.4, p<.05$.

If we look at the within-condition priming, for the related condition there were no signs of an 
effect of SOA (both $p \mathrm{~s}>.39$; note that the error rates were very low). For the unrelated condition, there was an increasing linear trend, $F(1,3)=$ 16.91, $M S E=93.3, p<.05$, as well as a significant quadratic component, $F(1,3)=11.58$, $M S E=24.3, p<.05$.

\section{Different responses}

The ANOVA on the latency data only revealed a main effect of SOA, $F(3,9)=31.38, M S E=$ 125.6, $p<.001$ : This effect revealed a linear decreasing trend, $F(1,3)=70.42, \quad M S E=$ $164.1, p<.005$, whereas the quadratic component was not significant $(F<1)$.

The ANOVA on the error data did not reveal any significant effects (all $p$ s $>.15$ ).

The results of the present experiment are very clear. As deduced by the within-condition baseline condition, the robust masked repetition priming effect obtained in "same" responses is a combination of a facilitative effect and an inhibitory effect. This effect is probably orthographic and/ or sublexical in nature because the pattern of data is remarkably similar for word and nonword stimuli (see Table 1). For "different" responses, there are no signs of a repetition priming effect, and the only significant effect is that the longer the SOA the faster the responses.

The question now is whether a zero-contingency situation alters the pattern of facilitative/ inhibitory priming effects. Experiment 2 was the same as Experiment 1, except for the "different" trials (e.g., field-field-HOUSE vs. field-waterHOUSE).

\section{EXPERIMENT 2}

\section{Method}

\section{Participants}

Four well-trained participants took part in the experiment. They were the same as those in Experiment 1.

\section{Materials}

The materials were the same as those in Experiment 1. The only difference is that we manipulated the relationship between the probe and the prime rather than the relationship between the prime and the target. Note that this only affects "different" trials. That is, "same" trials were constructed in the same way as in Experiment 1 (e.g., related condition: otoñootoño-OTOÑO vs. unrelated condition: otoñoarena-OTONO). In contrast, for "different" trials we manipulated the relationship between the probe and the prime (e.g., related condition: falda-falda-OTONO vs. unrelated condition: falda-arena-OTOÑO).

\section{Procedure}

This was the same as that in Experiment 1, except that the 20 practice trials were also presented under a zero-contingency scenario. As in Experiment 1, each participant received a different, randomized order of trials across four sessions (640 trials on each session). The sessions took place one week after Experiment 1.

\section{Results and discussion}

Incorrect responses (3.5\% of trials) and reaction times less than 250 or greater than $1,500 \mathrm{~ms}$ $(0.06 \%$ of trials) were excluded from the latency data. The mean latencies for correct responses and error rates are presented in Table 2. The design was the same as that in Experiment $1 .^{2}$ As occurred in Experiment 1, the ANOVA on the latency data showed a significant Prime Type $\times$ SOA $\times$ Type of Response interaction, $F(3,9)$ $=188.1, M S E=31.3, p<.001$; therefore, we examined separately "same" and "different" trials. Lexical status did not interact with any of the other factors, and the main effect of lexicality approached significance, $F(1,3)=7.76, M S E=$ 50.0, $p=.069$. For that reason, we focus on prime type and SOA as factors, independently of lexical status.

\footnotetext{
${ }^{2}$ As in Experiment 1, session did not interact with any of the other factors (all $p s>.24$ ); in this experiment, the main effect of session was not significant in the RT analysis, $F(3,9)=1.18, M S E=224, p>.30$.
} 
Table 2. Mean response times and percentage of errors for word and pseudoword targets in Experiment 2

\begin{tabular}{|c|c|c|c|c|c|c|}
\hline \multirow[b]{2}{*}{ Responses } & & & \multicolumn{4}{|c|}{$S O A$} \\
\hline & & & $13 \mathrm{~ms}$ & $27 \mathrm{~ms}$ & $40 \mathrm{~ms}$ & $53 \mathrm{~ms}$ \\
\hline \multirow[t]{6}{*}{ Same } & \multirow[t]{3}{*}{ Words } & Related & $388(0.6)$ & $378(0.3)$ & $362(0.3)$ & $354(0.0)$ \\
\hline & & Unrelated & $389(0.3)$ & $397(1.2)$ & 421 (11.6) & 445 (27.2) \\
\hline & & Priming & $1(-0.3)$ & $19(0.9)$ & $55(11.3)$ & $91(27.2)$ \\
\hline & \multirow[t]{3}{*}{ Nonwords } & Related & $404(0.9)$ & $389(0.6)$ & $381(0.6)$ & $370(0.9)$ \\
\hline & & Unrelated & $406(0.3)$ & $412(1.9)$ & $425(7.1)$ & 448 (21.6) \\
\hline & & Priming & $2(-0.6)$ & $23(1.3)$ & $44(6.6)$ & $78(20.7)$ \\
\hline \multirow[t]{6}{*}{ Different } & \multirow[t]{3}{*}{ Words } & Related & $428(2.2)$ & $423(3.7)$ & $425(3.4)$ & $429(6.2)$ \\
\hline & & Unrelated & 433 (1.9) & $420(0.9)$ & 407 (1.9) & 397 (1.9) \\
\hline & & Priming & $5(-0.3)$ & $-4(-2.8)$ & $-19(-1.6)$ & $-32(-4.4)$ \\
\hline & \multirow[t]{3}{*}{ Nonwords } & Related & $434(0.9)$ & $424(2.2)$ & $424(1.9)$ & $429(4.1)$ \\
\hline & & Unrelated & 433 (1.6) & 421 (1.6) & 404 (1.9) & $397(0.9)$ \\
\hline & & Priming & $-1(0.7)$ & $-2(-0.6)$ & $-20(0.0)$ & $-32(-3.2)$ \\
\hline
\end{tabular}

Note: Zero-contingency scenario. SOA = stimulus onset asynchrony. Mean response times in ms. Percentages of errors in parentheses.

\section{Same responses}

The ANOVA on the RT data revealed a main effect of prime type, $F(1,3)=77.57, M S E=$ $376.4, p<.005$, and SOA, $F(3,9)=14.92$, $M S E=36.8, p<.01$. More important, the interaction between prime type and SOA was significant, $F(3,9)=120.69, M S E=49.2, p<.001$. This interaction reflected that the effect of prime type was significant at $\mathrm{SOAs}$ of $27 \mathrm{~ms}$ and greater: 13-ms SOA, $F<1$; 27-ms SOA, $F(1$, $3)=71.91, \quad M S E=27.2, \quad p<.005 ; \quad 40-\mathrm{ms}$ SOA, $F(1,3)=54.51, M S E=241.6, p<.005$; 53-ms SOA, $F(1,3)=189.19, M S E=168.4$, $p<.005$.

As occurred in Experiment 1, if we look at the within-condition baseline, for the related condition there was a decreasing linear trend, $F(1,3)=$ $230.11, M S E=21.27, p<.005$, whereas the quadratic component was not significant, $F(1,3)=2.55$, $M S E=20.6, p>.20$. Likewise, for the unrelated condition, there was an increasing linear trend, $F(1,3)=123.36, M S E=112.2, p<.005$, as well as a significant quadratic component, $F(1,3)=$ 68.67, $M S E=8.4, p<.005$.

The ANOVA on the error data revealed a main effect of prime type, $F(1,3)=13.74, M S E=$
83.5, $p<.05$, and SOA, $F(3,9)=19.23$, $M S E=26.0, p<.001$. More important, the interaction between prime type and prime duration was significant, $F(3,9)=19.62, M S E=$ 25.6, $p<.001$. This interaction reflected that the effect of prime type was not significant at the two shortest SOAs-13-ms SOA, $F(1,3)=3$, $M S E=0.33, p>.18 ; 27-\mathrm{ms}$ SOA, $F(1,3)=$ $3.39, M S E=2.23, p=.16$ - whereas it was significant (or marginally significant) at the two longest SOAs-40-ms SOA, $F(1,3)=7.07$, $M S E=44.6, p=.076$; 53-ms SOA, $F(1,3)=$ 20.59, $M S E=113.1, p<.05$.

If we look at the within-condition priming analysis, for the repeated condition there were no signs of an effect of SOA (both $p$ s > .35). For the unrelated condition, there was an increasing linear trend, $F(1,3)=19.75, M S E=132.5$, $p<.05$, as well as a significant quadratic component, $F(1,3)=24.02, M S E=16.0, p<.05$.

\section{Different responses}

The ANOVA on the latency data revealed a main effect of prime type, $F(1,3)=45.91, M S E=$ 56.1, $p<.01$, and SOA, $F(3,9)=9.33, M S E$ $=104.8, p<.005$. Unlike Experiment 1 , here 
the interaction between prime type and SOA was significant, $F(3,9)=17.99, M S E=58.4, p<$ .001. This interaction reflected that the effect of prime type was not significant at the 13- and 26ms SOAs (both $p$ s $>.20$ ), while it was significant at the longer SOAs: $40-\mathrm{ms} \mathrm{SOA}, F(1,3)=36.95$, $M S E=35.6, p<.01$; 53-ms SOA, $F(1,3)=$ 39.91, $M S E=104.3, p<.01$.

If we look at the within-condition baseline (see Table 2), for the repeated condition there were no signs of an effect of SOA $(p>.40)$. Interestingly, for the unrelated condition, the pattern was very similar to that in Experiment 1: There was a decreasing linear trend, $F(1,3)=8.33, M S E=$ 68.1, $p<.01$, as well as a significant quadratic component, $F(1,3)=11.37, M S E=$ 131.4, $p<.05$.

The ANOVA on the error data did not reveal any significant effects (all $p$ s $>.11$ ).

The results are again quite clear. For "same" responses, the pattern of data was remarkably similar to that in Experiment 1. For "different" responses, there was one basic difference: The effect of SOA occurred only for the related condition.

A combined ANOVA of Experiments 1 and 2 for "same" trials on the latency data revealed no signs of an interaction between prime type and experiment: Prime Type $\times$ Experiment interaction, $F(1,3)=1.06, M S E=27.5, p>.37$; Prime Type $\times \mathrm{SOA} \times$ Experiment interaction, $F(3,9)=1.53, M S E=16.5, p>.27$. Not surprisingly, a combined ANOVA of Experiments 1 and 2 for "different" responses on the latency data showed a significant Prime Type $\times$ SOA $\times$ Experiment interaction, $F(3,9)=6.64, M S E=$ 31.4, $p<.05$.

\section{GENERAL DISCUSSION}

The present experiments offer important insights on the facilitative/inhibitory processes underlying the masked priming same-different matching task via an incremental priming technique. The idea here is that the interpretation of priming effects "is better constrained by a double baseline approach than when only across-condition comparisons are made" (Jacobs et al., 1995, p. 1108). First, the repetition priming effects for "same" trials are a mixture of facilitative and inhibitory effects-as deduced from the withincondition baseline. Second, as predicted by the Bayesian Reader model, the pattern of data for "same" trials is very much alike under a predictive-contingency scenario and under a zero-contingency scenario, whereas the pattern of data changes for "different" trials (see Figures 1 and 2). Third, the obtained effects were remarkably similar for word and nonword stimuli (i.e., the underlying priming effects are orthographic rather than lexical in nature), consistent with Norris and Kinoshita's claims (see Norris \& Kinoshita, 2008). For simplicity, we examine the implications of the findings regardless of lexical status.

According to the Bayesian Reader model, for "same" trials (e.g., house-house-HOUSE vs. housewater-HOUSE), the related prime house provides evidence supporting the decision that the response is "same", while the unrelated prime water provides evidence supporting the decision that response is "different". This assumption predicts that the overall (across-conditions) priming effects should be a mixture of a facilitative priming effect and an inhibitory priming effect. This is exactly the pattern of data we obtained when using the within-condition baseline: For the related primes, there was a significant decrease of response times as a function of the SOA, whereas for the unrelated primes there was an increase of response times (and error rates) as a function of the SOA (see Figure 2). Note here, however, that the simulations on the Bayesian Reader model reported by Norris and Kinoshita (2008) only offered net priming effects (i.e., no information of facilitative/inhibitory effects was provided).

When we look at the across-condition priming effect (see Tables 1 and 2), the pattern of masked repetition priming effects for "same" responses was remarkably similar in the predictive-contingency experiment and in the zero-contingency experiment. Thus, these findings provide evidence supporting the Bayesian Reader account (see Kinoshita \& Norris, 2010), while they pose some problems for any account based on a "response 

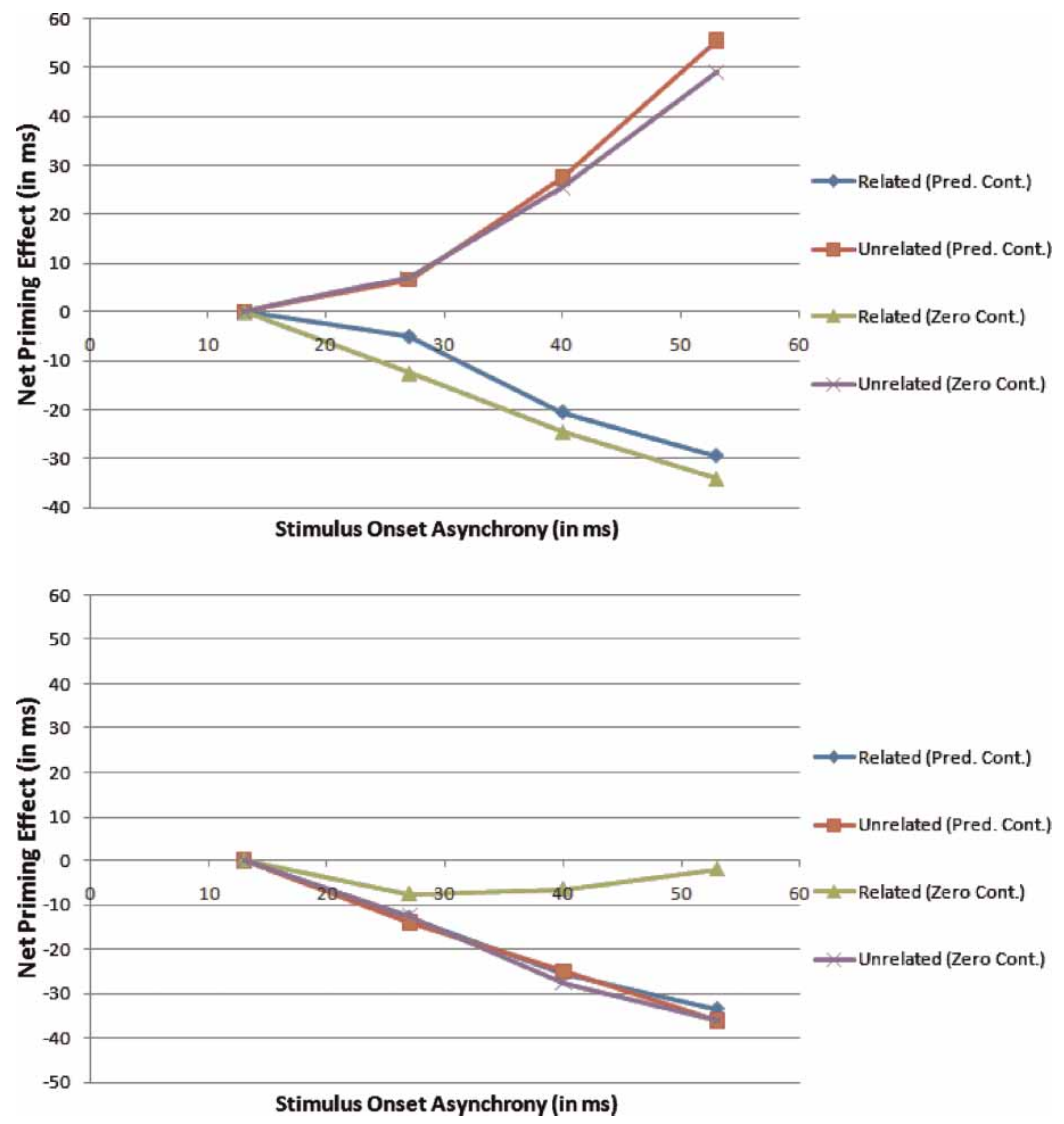

Figure 2. Net masked priming effects for "same" responses (top figure) and "different" responses (bottom figure) in the experiments. Net priming was computed as the difference (in ms) between the response time (RT) for each priming condition at the 13-ms SOA (stimulus onset asynchrony; i.e., the within-condition baseline) and the response time at the other SOAs (e.g., the net priming effect at the 53-ms $S O A$ would be the RT at the 53-ms SOA minus the RT at the 13-ms SOA). Note that positive values reflect inbibition, and negative values reflect facilitation, compared with the baseline. Data from word/nonword trials were combined. For "same" trials, the comparison was always between trials like house-house-HOUSE (related prime-target condition) and house-water-HOUSE (unrelated prime-target condition). For "different" trials, the comparison was between trials like field-house-HOUSE (related prime-target condition) versus field-water-HOUSE (unrelated prime-target condition) in the predictive-contingency experiment and between trials like field-field-HOUSE (related prime-target condition) versus field-water-HOUSE (unrelated prime-target condition) in the zero-contingency experiment. To view a colour version of this figure, please see the online issue of the Journal.

bias" dependent on probe/prime relationship. Keep in mind that a "response bias" account predicts a greater priming effect for "same" response in the predictive-contingency experiment: The reason is that, under this scenario, if the prime is similar to the probe, the response is necessarily "same". Of course, one might argue that just examining the across-condition priming effects does not provide the whole picture. If we look at the within-condition baseline, the pattern of facilitative priming effects for "same" responses was, if anything, slightly weaker (around $9 \mathrm{~ms}$ ) in the zero-contingency scenario than in the predictive-contingency scenario (see Figure 2). Thus, the observed pattern of data offers no empirical support for the "response bias" account.

What about masked priming effects in lexical decision versus same-different tasks? As stated in the introduction, masked priming effects in the lexical decision task are a combination of 
facilitative and inhibitory effects (e.g., around a 28-ms facilitation and a 19-ms inhibition in Giraudo \& Grainger's, 2001, Experiment 1, using an incremental priming technique; see Jacobs et al., 1995, for a similar pattern when manipulating the intensity of the prime). As indicated in the introduction, some critics of the same-different task argue that, for "same" trials, the target stimulus is already strongly activated by the probe. This implies that a related prime would presumably provide a slight additional activation. That is, in this view, the magnitude of priming effects with the same-different technique would be mostly inhibitory. The results of the present experiments are clear-cut (see Figure 2). We found a sizeable facilitative priming effect relative to the within-condition baseline (around 30-34 ms at the 53-ms SOA) - note that the magnitude of priming effects was quite similar in Experiments 1 and 2 (i.e., under predictive-contingency and zero-contingency scenarios). However, unlike lexical decision, in which the effects are facilitative rather than inhibitory (a 28-ms facilitative effects vs. an 18.5-ms inhibitory effect in the Giraudo \& Grainger, 2001, study), the nature of the priming effects in the masked priming same-different task tends to be more inhibitory rather than facilitative (see Figure 2). One reason for this pattern is that a prime that is unrelated with the target produces evidence supporting a "different" response, which must be overridden. Note that this component is absent in a lexical decision task. Thus, there may be an inherent inhibitory component which is specific to the same-different task. Simulations on a modified Bayesian Reader model are necessary to assess this possibility.

Let us now consider the findings for "different" trials. Under the predictive-contingency scenario (e.g., field-house-HOUSE vs. field-waterHOUSE), participants responded faster at longer SOAs, and this effect was not modulated by prime/ target relationship. The explanation, in terms of the Bayesian Reader model, is rather straightforward. Both the related prime house and the unrelated prime water contribute equally to a different decision. The longer the SOA, the greater the chances that that related/unrelated prime supports evidence of a "different" response, as actually occurred. Obviously, the predictions from the Bayesian Reader model differ under a zero-contingency scenario (e.g., fieldfield-HOUSE vs. field-water-HOUSE). Only the unrelated prime water contributes to a different decision. Consistent with this analysis, results showed that increasing the SOA produces faster responding but only for unrelated primes. With respect to related primes, the prime field may provide some evidence in support for a "same" response. Given that the correct response is "different", it is reasonable to assume that there would be some inhibitory effect. Indeed, if we look at the priming effect, we observed a significant (across-conditions) priming effect at the two longest SOAs (40and 53-ms SOAs). However, across-condition comparisons may not capture the full picture (see Jacobs et al., 1995). If we look at the within-condition baseline, the priming effect was not caused by an inhibition in the related condition; instead, it was caused by a facilitative effect in the unrelated condition. Simulations on the Bayesian Reader model would be necessary to examine whether the model can capture this pattern of results.

In general, the present findings provide empirical support for the Bayesian Reader model and pose some problems for those accounts that explain masked priming effects in the same-different task as a mere function of a "response bias". However, as an anonymous reviewer pointed out, there might be an inherent confound in the masked priming same-different task even under zero-contingency conditions. The reason is that, on "same" trials, the probe-prime-target sequence is composed of the same item repeated three times (e.g., house-house-HOUSE)-obviously, this cannot occur on "different" trials (e.g., field-fieldHOUSE or field-water-HOUSE) or on unrelated "same" trials (e.g., house-water-HOUSE). The argument is that if participants notice that every time the sequence of three stimuli is the same (or they fail to detect a change), then necessarily the response has to be "same". This strategy would predict that, for "same" trials, facilitation should grow in the related condition, whereas inhibition should develop in the unrelated condition as 
prime duration increases (i.e., the "response bias" strategy more easily applied with longer exposures of the prime). It would also correctly predict that, for "different" trials, the related condition (e.g., field-field-HOUSE) should be harder than the unrelated condition (e.g., field-water-HOUSE), given that there is a repetition in the former but not in the latter condition. (Note that a somehow parallel confound would occur in associative/ semantic priming experiments with the lexical decision task: If prime and target are related, the response must be "yes".) The point here is that, because of this potential confound, the use of masked primes in the same-different task would not be particularly informative. That is, one might as well use the standard, unprimed version of the same-different matching task. Although we do acknowledge some merit in the previous reasoning, it may not be entirely accurate. First, the above-cited strategy would predict a robust repetition priming effect with visible, unmasked primes even under a zero-contingency scenario (i.e., if the three items are the same, press "same"); however, Kinoshita and Norris (2010) failed to find a significant repetition priming effect under those conditions, which rules out the above-cited strategy. Second, leaving aside that masked priming effects can be obtained when the prime stimuli are not even detected (e.g., see Dehaene et al., 2001), and that primes may not be processed as separate objects from the target (Norris \& Kinoshita, 2008), masked repetition priming effects with the same-different task are negligible when the participants are presented with stimuli in an unfamiliar script (e.g., Arabic مقلوب مقلوب مقلوب مقاب no faster than مقلوب حارتس مقلوب see Perea et al., 2011): If participants were merely detecting whether the sequence of the three stimuli is the same or not, one would have expected a robust masked priming effect under these conditions. ${ }^{3}$
Furthermore, the masked priming same-different task offers valuable information that cannot be obtained in an unprimed version of the samedifferent matching task. For instance, one can obtain the time course of facilitation versus inhibition of masked priming effects, as shown in the present experiments, and one can perform some fine-tuned prime-target manipulations (e.g., "leet" priming as in VESZED-V35Z3DVESZED; see Perea et al., 2009). Of course, the masked priming same-different task is not free from potential shortcomings-like any other laboratory word identification task, but we believe that the masked priming same-different task is a valuable tool to make inferences on how the brain processes linguistic and nonlinguistic stimuli.

To sum up, we have demonstrated that masked priming effects with the same-different task are a combination of facilitative and inhibitory effects. The pattern of data for "same" responses was quite similar when the probe/prime relationship was diagnostic of the response to the target (predictive-contingency scenario) and when the prime/probe relationship was not diagnostic of the response to the target (zero-contingency scenario). Finally, the present experiments demonstrate once more the relevance of psychophysical studies for masked priming researchers, as anticipated by Grainger and colleagues (e.g., Grainger \& Frenck-Mestre, 1998; Jacobs et al., 1995; Ziegler et al., 2000).

Original manuscript received 9 December 2011 Accepted revision received 17 February 2011 First published online 9 July 2011

\section{REFERENCES}

Bowers, J. (2010a). Does masked and unmasked priming reflect Bayesian inference as implemented in the

\footnotetext{
${ }^{3}$ In addition, the only way the participants know that a given item has been repeated three times is once they have actually made the same-different decision. That is, in "different" trials under zero contingency, the probe and the prime are always the same until the participant encounters the different stimulus. What happens here is that participants in the masked priming same-different task are able to tell whether probe and target are the same or not. Note here that Norris and Kinoshita (2010) have already demonstrated that prime diagnosticity is relevant with visible, unmasked primes, whereas it does not play a role with masked primes.
} 
Bayesian Reader? European Journal of Cognitive Psychology, 22, 779-797.

Bowers, J. (2010b). What are the Bayesian constraints in the Bayesian reader? Reply to Norris and Kinoshita (2010). European Journal of Cognitive Psychology, 22, 1270-1273.

Davis, C. J., \&Perea, M. (2005). BuscaPalabras: A program for deriving orthographic and phonological neighborhood statistics and other psycholinguistic indices in Spanish. Behavior Research Methods, 37, 665-671.

Dehaene, S., Naccache, L., Cohen, L., Le Bihan, D., Mangin, J.-F., Poline, J.-B., et al. (2001). Cerebral mechanisms of word masking and unconscious repetition priming. Nature Neuroscience, 4, 752-758.

Duñabeitia, J. A., Kinoshita, S., Carreiras, M., \& Norris, D. (2011). Is morpho-orthographic decomposition purely orthographic? Evidence from masked priming in the same-different task. Language and Cognitive Processes, 26, 509-529.

Forster, K. I., \& Forster, J. C. (2003). DMDX: A Windows display program with millisecond accurary. Behavior Research Methods, Instruments, and Computers, 35, 116-124.

Frost, R., Ahissar, M., Gottesman, R, \& Tayeb, S. (2003). Are phonological effects fragile? The effect of luminance and exposure duration on form priming and phonological priming. Journal of Memory and Language, 48, 346-378.

García-Orza, J., \& Perea, M. (2011). Position coding in two-digit Arabic numbers: Evidence from number decision and same-different tasks. Experimental Psychology, 58, 85-91.

García-Orza, J., Perea, M., \& Muñoz, S. (2010). Are transposition effects specific to letters? Quarterly Journal of Experimental Psychology, 63, 1603-1618.

Giraudo, H., \& Grainger, J. (2001). Priming complex words: Evidence for supralexical representation of morphology. Psychonomic Bulletin E Review, 8, 127-131.

Grainger, J., \& Frenck-Mestre, C. (1998). Masked translation priming in bilinguals. Language and Cognitive Processes, 13, 601-623.

Jacobs, A. M., Grainger, J., \& Ferrand, L. (1995). The incremental priming technique: A method for determining within-condition priming effects. Perception E Psychophysics, 57, 1101-1110.

Kinoshita, S., Castles, A., \& Davis, C. (2009). The role of neighbourhood density in transposed-letter priming. Language and Cognitive Processes, 24, 506-526.
Kinoshita, S., \& Kaplan, L. (2008). Priming of abstract letter identities in the letter match task. Quarterly Journal of Experimental Psychology, 61, 1873-1885.

Kinoshita, S., \& Lagoutaris, S. (2010). Priming by NUMB3R5 does not involve top-down feedback. Journal of Experimental Psychology: Learning, Memory and Cognition, 36, 1422-1440.

Kinoshita, S., \& Norris, D. (2009). Transposed-letter priming of pre-lexical orthographic representations. Journal of Experimental Psychology: Learning, Memory and Cognition, 35, 1-18.

Kinoshita, S., \& Norris, D. (2010). Masked priming effect reflects evidence accumulated by the prime. Quarterly Journal of Experimental Psychology, 63, 194-204.

Kinoshita, S., \& Norris, D. (2011). Does the familiaritybias hypothesis explain why there is no masked priming for "NO" decisions? Memory \& Cognition, 39, 319-334.

Norris, D., \& Kinoshita, S. (2008). Perception as evidence accumulation and Bayesian inference: Insights from masked priming. Journal of Experimental Psychology: General, 137, 433-455.

Norris, D., \& Kinoshita, S. (2010). Explanation versus accommodation: Reply to Bowers (2010). European Journal of Cognitive Psychology, 22, 1261-1269.

Perea, M., Abu Mallouh, R., García-Orza, J., \& Carreiras, M. (2011). Masked priming effects are modulated by expertise in the script. Quarterly Journal of Experimental Psychology. 64, 902-919.

Perea, M., \& Acha, J. (2009). Does letter position coding depend on consonant/vowel status? Evidence with the masked priming technique. Acta Psychologica, 130, 127-137.

Perea, M., Duñabeitia, J. A, Pollatsek, A., \& Carreiras, M. (2009). Does the brain regularize digits and letters to the same extent? Quarterly Journal of Experimental Psychology, 62, 1881-1888.

Perea, M., \& Gotor, A. (1997). Associative and semantic priming effects occur at very short SOAs in lexical decision and naming. Cognition, 62, 223-240.

Peressotti, F., \& Grainger, J. (1999). The role of letter identity and letter position in orthographic priming. Perception E Psychophysics, 61, 691-706.

Ziegler, J., Ferrand, L., Jacobs, A. M., Rey, A., \& Grainger, J. (2000). Visual and phonological codes in letter and word recognition: Evidence from incremental priming. Quarterly Journal of Experimental Psychology, 53A, 671-692. 